\title{
LCSTS: A Large Scale Chinese Short Text Summarization Dataset
}

\author{
Baotian Hu Qingcai Chen Fangze Zhu \\ Intelligent Computing Research Center \\ Harbin Institute of Technology, Shenzhen Graduate School \\ \{baotianchina, qingcai.chen, zhufangze123\}@gmail.com
}

\begin{abstract}
Automatic text summarization is widely regarded as the highly difficult problem, partially because of the lack of large text summarization data set. Due to the great challenge of constructing the large scale summaries for full text, in this paper, we introduce a large corpus of Chinese short text summarization dataset constructed from the Chinese microblogging website Sina Weibo, which is released to the public ${ }^{1}$. This corpus consists of over 2 million real Chinese short texts with short summaries given by the author of each text. We also manually tagged the relevance of 10,666 short summaries with their corresponding short texts. Based on the corpus, we introduce recurrent neural network for the summary generation and achieve promising results, which not only shows the usefulness of the proposed corpus for short text summarization research, but also provides a baseline for further research on this topic.
\end{abstract}

\section{Introduction}

Nowadays, individuals or organizations can easily share or post information to the public on the social network. Take the popular Chinese microblogging website (Sina Weibo) as an example, the People's Daily, one of the media in China, posts more than tens of weibos (analogous to tweets) each day. Most of these weibos are wellwritten and highly informative because of the text length limitation (less than140 Chinese characters). Such data is regarded as naturally annotated web resources (Sun, 2011). If we can mine these high-quality data from these naturally annotated web resources, it will be beneficial to the research that has been hampered by the lack of data.

\footnotetext{
${ }^{1}$ http://icrc.hitsz.edu.cn/Article/show/139.html
}

【江西高考被曝替考有关考生已被警方控制】 人日报记者吴齐强消息，江西高考被曝光 替考, 7日中午江西省教育厅发布消息称，接到有人组织替考的举报后，江西省教育厅、 江西省教育考试院立即部署南昌市教育考试院，联合南昌市警方开展调查核实，有关考生 已被警方控制。调查进展情况将及时向社会公布。

Figure 1: A Weibo Posted by People's Daily.

In the Natural Language Processing (NLP) community, automatic text summarization is a hot and difficult task. A good summarization system should understand the whole text and re-organize the information to generate coherent, informative, and significantly short summaries which convey important information of the original text (Hovy and Lin, 1998), (Martins, 2007). Most of traditional abstractive summarization methods divide the process into two phrases (Bing et al., 2015). First, key textual elements are extracted from the original text by using unsupervised methods or linguistic knowledge. And then, unclear extracted components are rewritten or paraphrased to produce a concise summary of the original text by using linguistic rules or language generation techniques. Although extensive researches have been done, the linguistic quality of abstractive summary is still far from satisfactory. Recently, deep learning methods have shown potential abilities to learn representation (Hu et al., 2014; Zhou et al., 2015) and generate language (Bahdanau et al., 2014; Sutskever et al., 2014) from large scale data by utilizing GPUs. Many researchers realize that we are closer to generate abstractive summarizations by using the deep learning methods. However, the publicly available and high-quality large scale summarization data set is still very rare and not easy to be constructed manually. For example, the popular document summarization dataset DUC $^{2}$, TAC $^{3}$ and TREC ${ }^{4}$ have only hundreds of human written English text summarizations. The problem is even worse for Chinese. In this pa-

\footnotetext{
${ }^{2} \mathrm{http}: / /$ duc.nist.gov/data.html

${ }^{3}$ http://www.nist.gov/tac/2015/KBP/

${ }^{4}$ http://trec.nist.gov/
} 


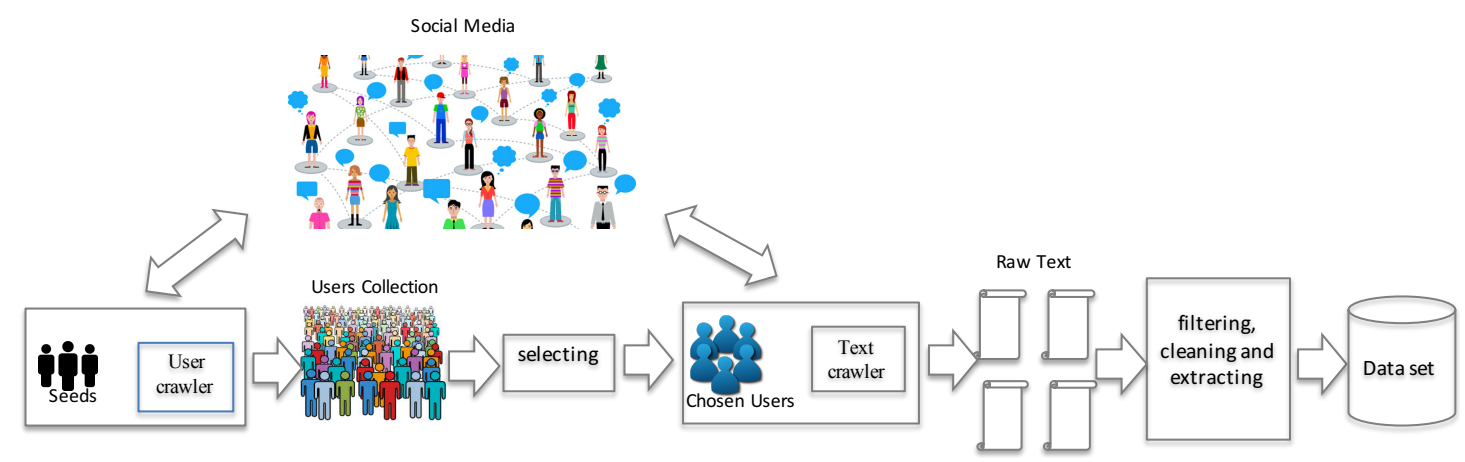

Figure 2: Diagram of the process for creating the dataset.

per, we take one step back and focus on constructing LCSTS, the Large-scale Chinese Short Text Summarization dataset by utilizing the naturally annotated web resources on Sina Weibo. Figure 1 shows one weibo posted by the People's Daily. In order to convey the import information to the public quickly, it also writes a very informative and short summary (in the blue circle) of the news. Our goal is to mine a large scale, high-quality short text summarization dataset from these texts.

This paper makes the following contributions: (1) We introduce a large scale Chinese short text summarization dataset. To our knowledge, it is the largest one to date; (2) We provide standard splits for the dataset into large scale training set and human labeled test set which will be easier for benchmarking the related methods; (3) We explore the properties of the dataset and sample 10,666 instances for manually checking and scoring the quality of the dataset; (4) We perform recurrent neural network based encoder-decoder method on the dataset to generate summary and get promising results, which can be used as one baseline of the task.

\section{Related Work}

Our work is related to recent works on automatic text summarization and natural language processing based on naturally annotated web resources, which are briefly introduced as follows.

Automatic Text Summarization in some form has been studied since 1950. Since then, most researches are related to extractive summarizations by analyzing the organization of the words in the document (Nenkova and McKeown, 2011) (Luhn, 1998); Since it needs labeled data sets for supervised machine learning methods and labeling dataset is very intensive, some researches focused on the unsupervised methods (Mihalcea, 2004). The scale of existing data sets are usually very small (most of them are less than 1000). For example, DUC2002 dataset contains 567 documents and each document is provided with two 100 -words human summaries. Our work is also related to the headline generation, which is a task to generate one sentence of the text it entitles. Colmenares et.al construct a 1.3 million financial news headline dataset written in English for headline generation (Colmenares et al., 2015). However, the data set is not publicly available.

Naturally Annotated Web Resources based Natural Language Processing is proposed by Sun (Sun, 2011). Naturally Annotated Web Resources is the data generated by users for communicative purposes such as web pages, blogs and microblogs. We can mine knowledge or useful data from these raw data by using marks generated by users unintentionally. Jure et.al track 1.6 million mainstream media sites and blogs and mine a set of novel and persistent temporal patterns in the news cycle (Leskovec et al., 2009). Sepandar et.al use the users' naturally annotated pattern 'we feel' and ' $i$ feel' to extract the 'Feeling' sentence collection which is used to collect the world's emotions. In this work, we use the naturally annotated resources to construct the large scale Chinese short text summarization data to facilitate the research on text summarization.

\section{Data Collection}

A lot of popular Chinese media and organizations have created accounts on the Sina Weibo. They use their accounts to post news and information. These accounts are verified on the Weibo and labeled by a blue ' $V$ '. In order to guarantee the quality of the crawled text, we only crawl the verified organizations' weibos which are more likely to be clean, formal and informative. There are a lot of human intervention required in each step. The process of the data collection is shown as Figure 2 and 
summarized as follows:

1) We first collect 50 very popular organization users as seeds. They come from the domains of politic, economic, military, movies, game and etc, such as People's Daily, the Economic Observe press, the Ministry of National Defense and etc. 2) We then crawl fusers followed by these seed users and filter them by using human written rules such as the user must be blue verified, the number of followers is more than 1 million and etc. 3) We use the chosen users and text crawler to crawl their weibos. 4) we filter, clean and extract (short text, summary) pairs. About 100 rules are used to extract high quality pairs. These rules are concluded by 5 peoples via carefully investigating of the raw text. We also remove those paris, whose short text length is too short (less than 80 characters) and length of summaries is out of $[10,30]$.

\section{Data Properties}

The dataset consists of three parts shown as Table 1 and the length distributions of texts are shown as Figure 3.

Part I is the main content of LCSTS that contains 2,400,591 (short text, summary) pairs. These pairs can be used to train supervised learning model for summary generation.

Part II contains the 10,666 human labeled (short text, summary) pairs with the score ranges from 1 to 5 that indicates the relevance between the short text and the corresponding summary. ' 1 ' denotes " the least relevant" and ' 5 ' denotes "the most relevant". For annotating this part, we recruit 5 volunteers, each pair is only labeled by one annotator. These pairs are randomly sampled from Part I and are used to analysize the distribution of pairs in the Part I. Figure 4 illustrates examples of different scores. From the examples we can see that pairs scored by 3, 4 or 5 are very relevant to the corresponding summaries. These summaries are highly informative, concise and significantly short compared to original text. We can also see that many words in the summary do not appear in the original text, which indicates the significant difference of our dataset from sentence compression datasets. The summaries of pairs scored by 1 or 2 are highly abstractive and relatively hard to conclude the summaries from the short text. They are more likely to be headlines or comments instead of summaries. The statistics show that the percent of score 1 and 2 is less than $20 \%$ of the

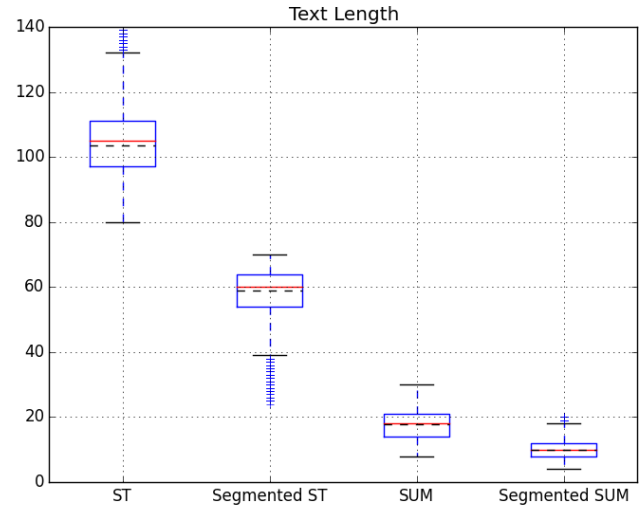

Figure 3: Box plot of lengths for short text(ST), segmented short text(Segmented ST), summary(SUM) and segmented summary(Segmented SUM). The red line denotes the median, and the edges of the box the quartiles.

data, which can be filtered by using trained classifier.

Part III contains 1,106 pairs. For this part, 3 annotators label the same 2000 texts and we extract the text with common scores. This part is independent from Part I and Part II. In this work, we use pairs scored by 3, 4 and 5 of this part as the test set for short text summary generation task.

\begin{tabular}{|c|c|c|}
\hline Part I & \multicolumn{2}{|c|}{$2,400,591$} \\
\hline \multirow{4}{*}{ Part II } & Number of Pairs & 10,666 \\
\cline { 2 - 3 } & Human Score 1 & 942 \\
\cline { 2 - 3 } & Human Score 2 & 1,039 \\
\cline { 2 - 3 } & Human Score 3 & 2,019 \\
\cline { 2 - 3 } & Human Score 4 & 3,128 \\
\cline { 2 - 3 } & Human Score 5 & 3,538 \\
\hline \multirow{5}{*}{ Part III } & Number of Pairs & 1,106 \\
\cline { 2 - 3 } & Human Score 1 & 165 \\
\cline { 2 - 3 } & Human Score 2 & 216 \\
\cline { 2 - 3 } & Human Score 3 & 227 \\
\cline { 2 - 3 } & Human Score 4 & 301 \\
\cline { 2 - 3 } & Human Score 5 & 197 \\
\hline
\end{tabular}

Table 1: Data Statistics

\section{Experiment}

Recently, recurrent neural network (RNN) have shown powerful abilities on speech recognition (Graves et al., 2013), machine translation (Sutskever et al., 2014) and automatic dialog response (Shang et al., 2015). However, there is rare research on the automatic text summarization by using deep models. In this section, we use RNN as encoder and decoder to generate the summary of short text. We use the Part I as the training set 
Short Text: 水利部水资源司司长陈明忠今日在新闻发布会上透露, 根据刚 刚完成的水资源管理制度的考核, 有部分省接近了红线的指标, 有部分省 超过红线的指标。在一些超过红线的地方, 将对一些取用水项目进行区域 的限批, 严格地进行水资源论证和取水许可的批准。

Mingzhong Chen, the Chief Secretary of the Water Devision of the Ministry of Water Resources, revealed today at a press conference, according to the justcompleted assessment of water resources management system, some provinces are closed to the red line indicator, some provinces are over the red line indicator. In some places over the red line, It will enforce regional approval restrictions on some water projects, implement strictly water resources assessment and the approval of water licensing.

Summarization: 部分省超过年度用水红线指标 取水项目将被限批

Some provinces exceeds the red line indicator of annual water using, some water project will be. limited approved

Human Score: 5

Short Text: 各团购网站移动端销售额占比均在 $30 \%$ 以下, 用户通过PC端购物 习惯短时间内难以转变。未来中国餐饮 020 市场, 移动端将成为餐饮 020 的 战略性发展方向, 也将由线上驱动转变为线下驱动。一二线城市面临增长 赛境, 三四线城市 020 市场蕴含机会。

Groupons' sales on mobile terminals are below 30 percent. User's preference of shopping through PCs can not be changed in the short term. In the future Chinese 020 catering market, mobile terminals will become the strategic development direction. And also, it will become off-line driving from on-line driving. The first and second tier cities are facing growth difficulties. However, $\mathrm{O} 2 \mathrm{O}$ market in the third and fourth tier cities contains opportunities.

Summarization: 移动端成餐饮 $\mathrm{O} 2 \mathrm{O}$ 的战略性发展方向

The mobile terminals will become catering's strategic development direction.

Human Score: 4

Short Text: 7月百城住宅新建住宣平均价格为 10347 元/平方米, 环比上涨 $0.87 \%$, 自去年 6 月以来连续 14 个月环比上涨。其中, 广州、北京、深圳、南 京胀幅均超过 $10 \%$ 。中原张大伟认为, 一二线城市因为集聚了过多资源, 房 价易涨难跌。

In July, 100-cities' average newly-built house prices is 10347 yuan per square, which rose $0.87 \%$. It rises for the $14^{\text {th }}$ consecutive month. Among them Guangzhou, Beijing, Shenzhen, Nanjing rise more than 10\%. Dawei Zhang, from Centaline Property Agency, said that because the first and second-tier city gathers too many resources, the price of house is likely to rise and hard to fall.

Summarization: 百城房价环比 “14连涨”一二线城市涨幅扩大

100-cities' house prices gain "14th consecutive rising", the first and second-tier cities rise more.

Human Score: 3

Short Text: 记者梳理发现, 2009年至今有8起福彩开奖延迟事件, 至少延迟 2 小时, 2014年5月6日第2014050期延迟开奖达4小时。8 起事件中福彩中心 对其中 3 起给出了回应, 理由有通讯故障及暴雨导致的数据上传延迟。另 5 起均未解释原因。

Reporters combed the information and found, from 2009 to now, there are at least 8 lottery delayed events and the delayed time are more than 2 hours. On May 6, 2014, the No. 2014050 delay more than 4 hours. The center of welfare lottery only respond to 3 of the 8 event. Their explanations are that a communications breakdown and heavy rain led to a data upload extension. There are no explanations for other 5 delay events.

Summarization:三问双色球开奖延迟: 开奖为何要等数据汇总?

Ask about the lottery delay third times: why lottery should wait data collection? Human Score: 2

Short Text: 商务部数据显示, 中国7月实际利用外资同比大幅下降 $16.95 \%$ 至 78.1 亿美元。外界有分析与近期官方对外资企业的密集反垄断调查有关。 沈丹阳回应指出, “不能与对外资的反垄断调查挂钩, 或者做其他没有根 据的联想”。

According to China's Ministry of Commerce, China's actually utilized foreign capital in July fell sharply about $16.95 \%$ to 7.81 billion dollars, comparing to last year. Analysis of the outside world believe that it is related to the recent official intensive antitrust investigation. Danyang Shen responded, "It can not be linked to the antitrust investigation of foreign investment, or do other unfounded association"

Summarization: 商务部表态反垄断：几个案子不会把外商吓回去

China's Ministry of Commerce respond to antitrust investigation: Several cases will not scare foreign investors away.

Human Score: 1

Figure 4: Five examples of different scores.

and the subset of Part III, which is scored by 3, 4 and 5 , as test set.

Two approaches are used to preprocess the data: 1) character-based method, we take the Chinese character as input, which will reduce the vocabulary size to 4,000. 2) word-based method, the

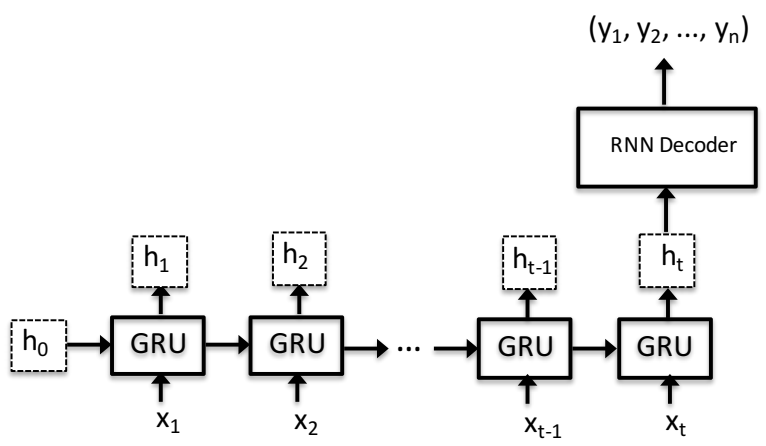

Figure 5: The graphical depiction of RNN encoder and decoder framework without context.

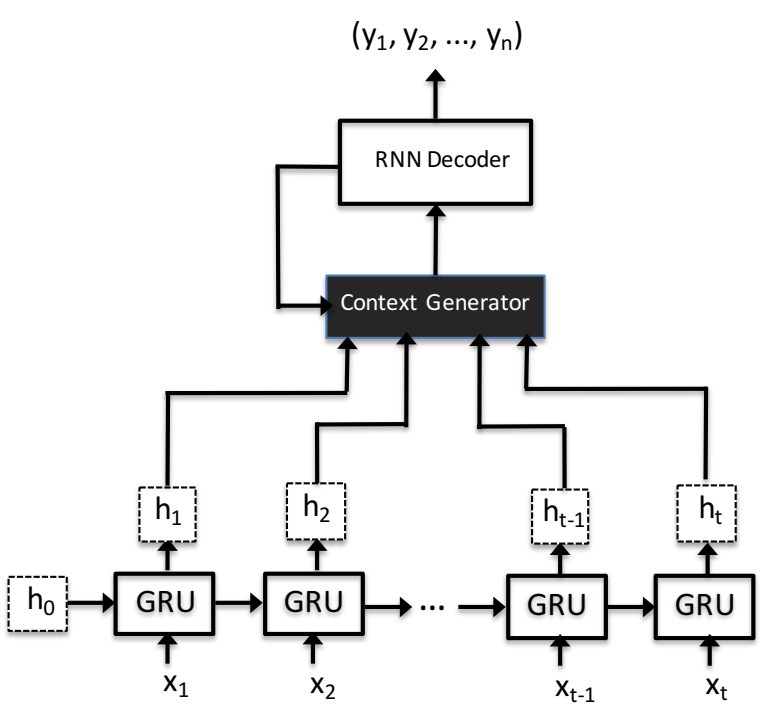

Figure 6: The graphical depiction of the RNN encoder and decoder framework with context.

text is segmented into Chinese words by using jieba $^{5}$. The vocabulary is limited to 50,000 . We adopt two deep architectures, 1) The local context is not used during decoding. We use the RNN as encoder and it's last hidden state as the input of decoder, as shown in Figure 5; 2) The context is used during decoding, following (Bahdanau et al., 2014), we use the combination of all the hidden states of encoder as input of the decoder, as shown in Figure 6. For the RNN, we adopt the gated recurrent unit (GRU) which is proposed by (Chung et al., 2015) and has been proved comparable to LSTM (Chung et al., 2014). All the parameters (including the embeddings) of the two architectures are randomly initialized and ADADELTA (Zeiler, 2012) is used to update the learning rate. After the model is trained, the beam search is used to generate the best summaries in the process of decoding and the size of beam is set to 10 in our experiment.

\footnotetext{
${ }^{5}$ https://pypi.python.org/pypi/jieba/
} 


\begin{tabular}{|c|c|c|c|c|}
\hline model & data & R-1 & R-2 & R-L \\
\hline \multirow{2}{*}{ RNN } & Word & 0.043 & 0.025 & 0.043 \\
\cline { 2 - 5 } & Char & 0.061 & 0.028 & 0.057 \\
\hline \multirow{2}{*}{ RNN context } & Word & 0.087 & 0.054 & 0.085 \\
\cline { 2 - 5 } & Char & $\mathbf{0 . 1 0 8}$ & $\mathbf{0 . 0 7 3}$ & $\mathbf{0 . 1 0 7}$ \\
\hline
\end{tabular}

Table 2: The experiment result: "Word" and "Char" denote the word-based and characterbased input respectively.

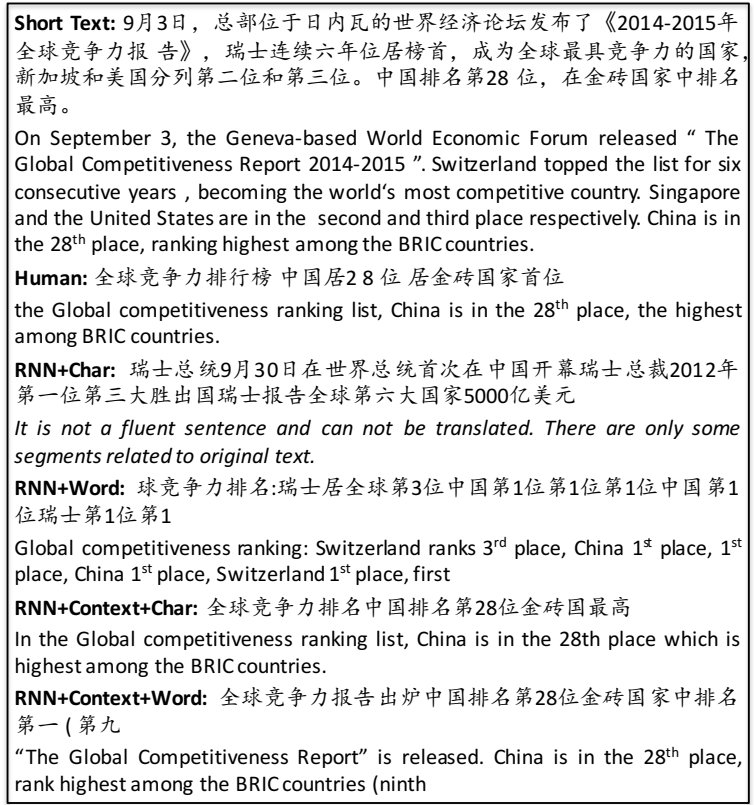

Figure 7: An example of the generated summaries.

For evaluation, we adopt the ROUGE metrics (Lin, 2004) proposed by (Lin and Hovy, 2003), which have been proved strongly correlated with human evaluations. ROUGE-1, ROUGE2 and ROUGE-L are used. All the models are trained on the GPUs tesla M2090 for about one week.Table 2 lists the experiment results. As we can see in Figure 7, the summaries generated by RNN with context are very close to human written summaries, which indicates that if we feed enough data to the RNN encoder and decoder, it may generate summary almost from scratch.

The results also show that the RNN with context outperforms RNN without context on both character and word based input. This result indicates that the internal hidden states of the RNN encoder can be combined to represent the context of words in summary. And also the performances of the character-based input outperform the wordbased input. As shown in Figure 8, the summary generated by RNN with context by inputing the character-based short text is relatively good, while the the summary generated by RNN with context

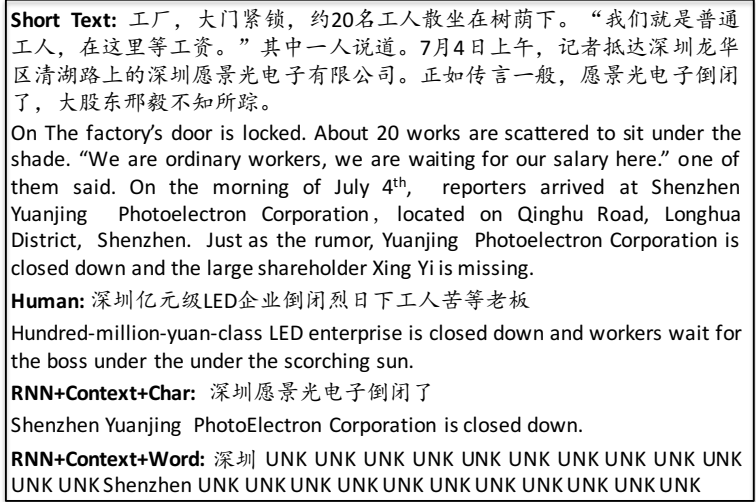

Figure 8: An example of the generated summaries with UNKs.

on word-based input contains many UNKs. This may attribute to that the segmentation may lead to many UNKs in the vocabulary and text such as the person name and organization name. For example, “愿景光电子” is a company name which is not in the vocabulary of word-based RNN, the RNN summarizer has to use the UNKs to replace the “愿景光电子” in the process of decoding.

\section{Conclusion and Future Work}

We constructed a large-scale Chinese short text summarization dataset and performed RNN-based methods on it, which achieved some promising results. This is just a start of deep models on this task and there is much room for improvement. We take the whole short text as one sequence, this may not be very reasonable, because most of short texts contain several sentences. A hierarchical RNN (Li et al., 2015) is one possible direction. The rare word problem is also very important for the generation of the summaries, especially when the input is word-based instead of character-based. It is also a hot topic in the neural generative models such as neural translation machine(NTM) (Luong et al., 2014), which can benefit to this task. We also plan to construct a large document summarization data set by using naturally annotated web resources.

\section{Acknowledgments}

This work is supported by National Natural Science Foundation of China: 61473101, 61173075 and 61272383, Strategic Emerging Industry Development Special Funds of Shenzhen: JCYJ20140417172417105, JCYJ20140508161040764 and JCYJ20140627163809422. We thank to Baolin Peng, Lin Ma, Li Yu and the anonymous reviewers for their insightful comments. 


\section{References}

Dzmitry Bahdanau, Kyunghyun Cho, and Yoshua Bengio. 2014. Neural machine translation by jointly learning to align and translate. CoRR, abs/1409.0473.

Lidong Bing, Piji Li, Yi Liao, Wai Lam, Weiwei Guo, and Rebecca Passonneau. 2015. Abstractive multidocument summarization via phrase selection and merging. In Proceedings of the ACL-IJCNLP, pages 1587-1597, Beijing, China, July. Association for Computational Linguistics.

Junyoung Chung, Çaglar Gülçehre, KyungHyun Cho, and Yoshua Bengio. 2014. Empirical evaluation of gated recurrent neural networks on sequence modeling. CoRR, abs/1412.3555.

Junyoung Chung, Çaglar Gülçehre, KyungHyun Cho, and Yoshua Bengio. 2015. Gated feedback recurrent neural networks. CoRR, abs/1502.02367.

Carlos A. Colmenares, Marina Litvak, Amin Mantrach, and Fabrizio Silvestri. 2015. Heads: Headline generation as sequence prediction using an abstract feature-rich space. In Proceddings of 2015 Conference of the North American Chapter of the Association for Computational Linguistics-Human Language Technologies (NAACL HLT 2015).

Alex Graves, Abdel-rahman Mohamed, and Geoffrey E. Hinton. 2013. Speech recognition with deep recurrent neural networks. CoRR, abs/1303.5778.

Eduard Hovy and Chin-Yew Lin. 1998. Automated text summarization and the summarist system. In Proceedings of a Workshop on Held at Baltimore, Maryland: October 13-15, 1998, TIPSTER '98, pages 197-214, Stroudsburg, PA, USA. Association for Computational Linguistics.

Baotian $\mathrm{Hu}$, Zhengdong Lu, Hang Li, and Qingcai Chen. 2014. Convolutional neural network architectures for matching natural language sentences. In Advances in Neural Information Processing Systems 27, pages 2042-2050. Curran Associates, Inc.

Jure Leskovec, Lars Backstrom, and Jon Kleinberg. 2009. Meme-tracking and the dynamics of the news cycle. In Proceedings of the 15th ACM SIGKDD International Conference on Knowledge Discovery and Data Mining, KDD '09, pages 497-506.

Jiwei Li, Minh-Thang Luong, and Dan Jurafsky. 2015. A hierarchical neural autoencoder for paragraphs and documents. In Proceedings of $A C L$.
Chin-Yew Lin and E.H. Hovy. 2003. Automatic evaluation of summaries using n-gram co-occurrence statistics. In Proceedings of 2003 Language Technology Conference (HLT-NAACL 2003), Edmonton, Canada.

Chin-Yew Lin. 2004. Rouge: A package for automatic evaluation of summaries. In In Proceedings of Workshop on Text Summarization Branches Out, Post-Conference Workshop of ACL 2004, Barcelona, Spain.

H. P. Luhn. 1998. The automatic creation of literature abstracts. IBM Journal of Research and Development, 2(2):159-165.

Thang Luong, Ilya Sutskever, Quoc V. Le, Oriol Vinyals, and Wojciech Zaremba. 2014. Addressing the rare word problem in neural machine translation. CoRR, abs/1410.8206.

Dipanjan Das Andr F.T. Martins. 2007. A survey on automatic text summarization. Technical report, CMU.

Rada Mihalcea. 2004. Graph-based ranking algorithms for sentence extraction, applied to text summarization. In Proceedings of the 42nd Annual Meeting of the Association for Computational Linguistics, companion volume, Spain.

Ani Nenkova and Kathleen McKeown. 2011. Automatic summarization. Foundations and Trend in Information Retrieval, 5(2-3):103-233.

Lifeng Shang, Zhengdong Lu, and Hang Li. 2015. Neural responding machine for short-text conversation. CoRR, abs/1503.02364.

Mao Song Sun. 2011. Natural language procesing based on naturaly annotated web resources. Journal of Chinese Information Processing, 25(6):26-32.

Ilya Sutskever, Oriol Vinyals, and Quoc V. V Le. 2014. Sequence to sequence learning with neural networks. In Advances in Neural Information Processing Systems 27, pages 3104-3112.

Matthew D. Zeiler. 2012. ADADELTA: an adaptive learning rate method. CoRR, abs/1212.5701.

Xiaoqiang Zhou, Baotian Hu, Qingcai Chen, Buzhou Tang, and Xiaolong Wang. 2015. Answer sequence learning with neural networks for answer selection in community question answering. In Proceedings of the ACL-IJCNLP, pages 713-718, Beijing, China, July. Association for Computational Linguistics. 